\title{
Psychometric Properties of the Chinese Version of the Eating Attitudes Test (EAT-26) in Young Female Patients with Eating Disorders in Mainland China
}

Qing Kang ${ }^{1}$, Raymond C. K. Chan ${ }^{2,3}$, Xiaoping Li ${ }^{4}$, Jon Arcelus ${ }^{5}$, Ling Yue ${ }^{1}$, Jiabin Huang ${ }^{1}$, Lian $\mathrm{Gu}^{1}$, Qing Fan ${ }^{1}$, Haiyin Zhang ${ }^{1}$, Zeping Xiao ${ }^{1 \#} \& J_{u e}$ Chen $^{1 \#}$

${ }^{1}$ Department of Clinical Psychology, Shanghai Mental Health Center, Shanghai Jiao Tong University School of Medicine, Shanghai, China

${ }^{2}$ TranslationalNeuropsychology and Applied Cognitive Neuroscience Laboratory, Shanghai Mental Health Center, Shanghai Jiao Tong University School of Medicine, Shanghai, China

${ }^{3}$ Neuropsychology and Applied Cognitive Neuroscience Laboratory, Key Laboratory of Mental Health, Institute of Psychology, Chinese Academy of Sciences, Beijing, China

${ }^{4}$ Psychological Measurement Room, Shanghai Mental Health Center, Shanghai Jiao Tong University School of Medicine, Shanghai, China

${ }^{5}$ Institute of Mental Health, Faculty of Medicine \& Health Sciences, University of Nottingham, Nottingham, United Kingdom

\#: Corresponding authors: Zeping Xiao, Jue Chen

E-mail: Zeping Xiao,xiaozeping88@163.com, Department of Clinical Psychology, Shanghai Mental Health Center, Shanghai Jiao Tong University School of Medicine, 600 Wan Ping Nan Road, Shanghai, China

E-mail: Jue Chen, chenjue2088@163.com, Department of Clinical Psychology, Shanghai Mental Health Center, Shanghai Jiao Tong University School of Medicine, 600 Wan Ping Nan Road, Shanghai, China

This study was supported by grants from the Natural Science Foundation of Shanghai (16ZR1430400 to JC), the Scientific Technology Committee Foundation of Shanghai (16411965200 to JC) and the Key Characteristic Specialist Department of Shanghai Mental Health Center (No.2013-YJTSZK-02). 


\section{Abstract}

The study aimed to investigate the reliability and validity of the Chinese version of the EAT26among female adolescents and young adults in Mainland China. This scale was administered to 396 female EDs patients and 406 non-eating disorder healthy controls(HC), in addition 35HCcompleted a retest after a 4-week intervals. Tests for reliability, convergent validity and ROC analysis were performed to detect the psychometric properties. The EAT-26demonstrated good internal consistency(Cronbach alpha $=0.822 \sim 0.922)$, test-retest reliability $(\mathrm{ICC}=0.817)$, and convergent validity $(r=0.450 \sim 0.750)$. The ROC analysis showed that the cutoff 14 for AN and 15 for $\mathrm{BN}$ represented good compromises with approximate sensitivity $(0.66 \sim 0.68)$ and specificity $(0.85 \sim 0.86)$. Our findings provided evidence that the Chinese version of the EAT-26wasa psychometrically reliable and valid self-rating instrument for identifying people suffering from an ED in Mainland China. A clinical cutoff range between 14 and 15 could be used but caution should be exercise due to the low sensitivity of the tool.

Keywords:EAT-26; eating disorder; psychometric properties; cutoff, Mainland China 


\section{Introduction and aims}

Eating disorders (EDs) have been found to affect young females particularly (Hudson, Hiripi, Pope, \&Kessler, 2007) and to be common in developed countries(Dolan, 1991). In addition EDs and weigh concerns are becoming more prevalent in developing China(Huon, Qian, Oliver, \& Xiao, 2002; Lee, S.,\& Lee, A. M., 2000; Tong et al., 2014).

A number of assessment tools for EDs have been developed to be used in clinic and research settings and some of them have been translated in order to be used in China, such as the Eating Attitudes Test 26-item version (EAT-26) (Garner, Olmsted, Bohr, \& Garfinkel, 1982; Lee, Kwok, Liau, \& Leung, 2002), the Eating Disorder Examination (EDE) (Tong et al., 2011), or the Eating Disorder Inventory(EDI-1) (Garner, Olmstead, \& Polivy, 1983; Zhang, W. H., Zhang, D. R., \& Qian, 2006). Although the EDE and EDI-1 have demonstrated to have good validity and reliability among the Mainland Chinese EDs population(Tong et al., 2011; Zhang,\& Kong, 2004), they are limited by their length. This makes them unsuitable to be used in primary care settings. However, the EAT-26, a widely used self-report instrument, has already been used successfully to screen EDs patients in Hong Kong, China(Lee et al., 2002; Lee, S., \& Lee, A. M., 2000). The results of these studies showed differences in EDs symptomatology between people from Hong Kong and Mainland China, suggesting the need to re-establish the validity and reliability of EAT-26 in female EDs population in Mainland China.

The EAT-26(Garner et al., 1982) is one of the most empirically supported and oldest selfreport instruments used to identify people suffering from an ED. A clinical cutoff of 20 was initially established which was found to offer the best compromise between sensitivity(1.00) and 
specificity(0.91)(King, 1986). A more recent American study confirmed that this cutoff had a diagnostic accuracy rate of $90 \%$ with a similar specificity (0.94) but a lower sensitivity(0.77)(Mintz \& O'Halloran, 2000).Over the years a large body of literature has investigated the psychometric properties of the EAT-26 in different(primarily Western) cultures and among clinical and community samples(Garfinkel \& Newman, 2001; Al-Adawi, Dorvlo, Burke, Moosa, \& Al-Bahlani, 2002; Leeet al., 2002;Nunes, Camey, Olinto, \&Mari, 2005;Siervo, Boschi, Papa, Bellini, \&Falconi, 2005; Rivas, Bersabé, Jiménez,\&Berrocal, 2010).However, there are some inconsistencies regarding the cutoffs suggested by the findings of these studies. For example, an Italian study found that a clinical cutoff of 10 (instead of 20) was more appropriate to differentiate clinical from nonclinical people among the Italian population they studied (Siervoet al., 2005). These studies suggest that the clinical cut off may be influenced by the target population.

As research suggested that EDs are increasingly prevalent in Mainland China(Lee, S., \& Lee, A. M., 2000;Gail, Qian, Kylie, \& Guanglan, 2002;Tonget al., 2014), having a validated diagnostic tool for this population is important. In addition, as a recent study has demonstrated differences in the presentation of people with AN between Western and non-Western societies(Agüeraet al, 2017), these instruments need to be validated for the Chinese population. Lee et al (2002) proposed that a clinical cutoff of 20 would lead to an underestimation of atypical AN in community surveys in Hong Kong(Lee et al., 2002) and they found the mean of EAT-26 score(18.4) of AN patients was less than 20. However, Hong Kong is an international metropolis with similar socio-cultural values to western countries. This will indicated that the psychometric data provided by EDs samples in Hong Kong and other Western societies may not apply to Mainland Chinese EDs population. 
In view of this, the aim of the current study was to investigate the reliability, validity and clinical cutoff of the Chinese version of the EAT-26 (Lee et al., 2002; Lee et al., 2002) among female adolescents and young adults in Mainland China.

\section{Method}

\section{Participants and Procedure}

The clinical EDs group consisted of 396 female patients, including 157 adolescents(13 18 years old) and 239 adults(18 29 years old). Every patient recruited in the study was born and lived in Mainland China, assessed at the Shanghai Mental Health Center and fulfilled a diagnostic criteria for an eating disorder (DSM-IV-TR)(APA, 2000). The study took place at the Clinical Psychology Department of the Shanghai Mental Health Center(SMHC, Shanghai, China) between January 2003 to December 2015.

Using the DSM-IV-TR criteria, the clinical group consisted of 230 patients who full fielded diagnostic criteria for anorexia nervosa (AN), 84 for bulimia nervosa (BN), and 82 for Eating Disorders Not Otherwise Specified (EDNOS). In view of the new version of the DSM (DSM-5, APA, 2013) and as previous studies (Brewin, Baggott, Duggart, \& Arcelus, 2014) patients were rediagnosed using the new classification system, the total clinical sample consisted of 240 patients with AN, 84 with BN, 28 with binge eating disorder (BED), and 44 with other specified feeding or eating disorder (OSFED). The re-diagnosis under DSM-5 was performed by the first author (QK), and reviewed independently by another author (JC). Both of the diagnoses and re-diagnoses were established by licensed psychiatrists experienced in eating disorders. The detailed way of rediagnosing can be found in the supplementary material. 
The non-clinical healthy control (HC) group consisted of 406 females, including 184 adolescents(13 18 years old) and 222 adults (18 29 years old). They were recruited from middle schools $(n=72)$, high schools $(n=132)$, universities $(n=160)$ and jobholders $(n=42)$ between June 2003 to December 2015 in Shanghai, China via advertisements. Every participant from the HC group was used the Chinese version of the Mini-International Neuropsychiatric Interview (M.I.N.I.) (Pinninti, Madison, Musser, \&Rissmiller,2003; Si et al., 2009) to screen and confirm without an ED or any other psychiatric diagnosis.

Both groups, if agreed participation, completed a battery of questionnaires. The test-retest assessment was performed in the HC group only, as the EAT-26 scores in the EDs group could change during a 4-week intervals treatment, and influence the accuracy of the test-retest reliability. Finally $35 \mathrm{HC}$ voluntarily completed the EAT-26 twice at a 4-week intervals.

The study was approved by the Local Ethics Committees at the Shanghai Mental Health Center. A consent form was signed by participant above the age of 18 years and by the parents of those below 18 years, who agreed participation.

\section{Measures}

1. The Chinese version of the Eating Attitudes Test 26-item version (EAT-26) (Garner $e t$ al, 1982; Lee \&Lee, 1996)

The Eating Attitudes Test 26-item version (EAT-26)is a widely used self-rating questionnaire that assesses disturbed eating attitudes and behaviors (Garner et al., 1982). The answer for each item uses a 6-point likert score system (range $0=$ never to $5=$ always)which are grouped into 4-point format $(0=$ never, rarely and sometimes, $1=$ often, $2=$ usually, $3=$ always $)$, total scores can range from 
0 to 78. The Chinese version of theEAT-26was provided by Lee (Lee et al., 2000; Lee et al., 2002). As four different factor models of the EAT-26 have been reported in China (Leeet al., 2000) and even more models have been proposed in different cultures (Garfinkel et al., 2001), we used the EAT-26 total score rather than subscale scores to conduct the analysis.

2. The Chinese version of the Eating Disorder Inventory-I (EDI-I) (Garner, Olmstead, \& Polivy, 1983;Zhang, W. H., Zhang, D. R., \& Qian, Y., 2006)

The EDI-I is a64-item self-report instrument that is designed for the assessment of psychological and behavioral symptoms of $\mathrm{ED}$ (Garner et al.,1983). Each item is scored on a 3-point scale. The EDI-I has 8 subscales: Drive for thinness, Bulimia, Body dissatisfaction, Ineffectiveness, Perfectionism, Interpersonal distrust, Interoceptive awareness, and Maturity fears. The EDI-I was used to assess the convergent validity of the EAT-26 in our study. The Chinese version of the EDII has demonstrated good psychometric properties in clinical and non-clinical groups in Mainland China (Zhanget al., 2006). The Cronbach's alpha for the present ED and HC groups were 0.911 and 0.814 for the total scale, respectively.

3. The Chinese version of the Mini-International Neuropsychiatric Interview (M.I.N.I.) (Pinninti et al.,2003; Siet al., 2009)

The M.I.N.I. is a structured diagnostic interview schedule that was developed to assess the diagnoses of psychiatric patients (Pinnintiet al.,2003). The M.I.N.I. was used to screen for EDs and other psychiatric disorders among all the participants in the current study. The Chinese version of the M.I.N.I. has good validity and reliability according to SI et al. (2009) in Mainland China (Si et al., 2009). 


\section{Data Analysis}

The data were analyzed using SPSS 21.0(IBM Corporation, Armonk, New York, U.S.A.). Independent t-tests, analysis of variance (ANOVA) and analysis of covariance (ANCOVA) were used for continuous variables. Two-tailed significance levels were set at $p<0.05$. Bonferroni correction was applied when multiple comparisons occurred.

Cronbach's alpha and interclass correlation coefficient (ICC) over 4-week intervals were used to examine the internal consistency and test-retest reliability of the EAT-26, respectively. An ICC of at least 0.75 indicates an acceptable level (Weir, 2005).Pearson correlations with EDI-I total score were conducted to evaluate the convergent validity.

A receiver operating characteristic (ROC) analysis was calculated to assess the different cutoffs between sensitivity and specificity in discriminating ED patients from controls. Area under the curve (AUC) values, sensitivity, specificity, and the Youden index(YI) were reported in the text. An AUC value of $0.50 \sim 0.70$ is considered fair, an AUC of 0.70 0.90isgood, and an AUC>0.90 is excellent (Swets, 1988).

\section{Results}

\section{Demographical Characteristics of All Participants}

The demographic data are summarized in Table 1 and sTable 1. Compared with HC,EDs patients were older and had lower BMI (all $p<0.05$ ). When age was controlled for as a covariate, the ANCOVA also showed lower BMI in the EDs group $(p<0.05)$. 


\section{Differences in the Means of Total EAT-26 Scores by Group}

Participants in the EDs group had significantly higher EAT-26 total scores than did those in the HC group $(t=20.109, p<0.001)$. Bonferroni post hoc tests showed that the scores of most of the groups differed significantly from one another: Patients with BN had the highest scores, and $\mathrm{HC}$ controls had the lowest scores. Likewise, when the age was controlled as a covariate, the statistical difference in the total EAT-26 score among subgroups were in accordance with the above results: the BN group was the highest, and the HC group was the lowest(see Table 1).

\section{Reliability}

\section{Internal Consistency}

The internal consistency of the EAT-26 was assessed with Cronbach's alpha, and the results revealed satisfactory levels for all groups. Cronbach's alphas for the EAT-26 total scores for the EDs, AN, BN, BED,OSFED, and HC groups were 0.910, 0.915, 0.879, 0.919,0.822, and 0.830, respectively.

\section{Test-retest Reliability}

As stated above, $35 \mathrm{HC}$ completed the retest after 4-week intervals. At the first self-evaluation, the total EAT-26 score ranged from 0 to 19 (mean=5.43, $S D=5.19$ ). At the retest stage, it ranged from 0 to 22 (mean=4.83, SD=4.97). Test-retest reliability was good ( $\mathrm{ICC}=0.817, p<0.001)$. A paired-sample $t$ test showed no significant differences between the test and retest scores for the total EAT-26( $t=0.54, p=0.593)$. In addition, we compared the mean age, BMI, and total EAT-26 score 
between the respondents $(n=35)$ and non-respondents $(n=351)$ for the test-retest analysis, and the results showed no difference in these variables between the two groups (all $p>0.05$ ).

\section{Convergent Validity}

Convergent validity was analyzed with Pearson correlations between the total EAT-26 scores and EDI-I scores, and the results revealed significant moderate to large correlations in all groups (see sTable 2). The correlations between the total scores of EAT-26 and EDI-I for the EDs, AN, BN, BED, OSFED and HC groups were $0.744,0.743,0.567,0.450,0.661$ and 0.513 , respectively (all $p<0.001)$.

\section{ROC Analysis for the Sensitivity and Specificity of the EAT-26}

For the EDs group, according to the highest YI (0.524), the cutoff of 15 provided the best compromise between sensitivity (0.66) and specificity (0.86), and the AUC was 0.833 (95\% CI: 0.807, 0.806). For the AN and BN groups, the calculated optimal cutoffs were 14 and 15 , respectively. Summarily, the cutoff range from 14 to 15 yielded approximate sensitivity (0.66 0.68),specificity (0.85 0.86) and YI to differentiate EDs (including AN and BN) patients from non-clinical populations. The performance of other possible cutoffs reported in previous studies, ranging from 10 to 23 were presented in Table 2 (King, 1986; Al-Adawi et al., 2002; Rivas et al., 2010).

\section{Discussion}


The present study assessed the cross-cultural clinical utility of the Chinese version of the EAT26 among females with EDs in Mainland China. The results demonstrated that the Chinese version of the EAT-26 was a psychometrically reliable and valid self-report instrument for this population.

The reliability and validity analyses of the EAT-26 revealed sufficient internal consistency and convergent validity for the different ED sub-diagnoses. The test-retest reliability of this scale was very good. The result of sufficient internal consistency was consistent with the findings of previous studies (Lee et al., 2002; Nunes et al., 2005). The results also supported good convergent validity among female adolescents and young adults in Mainland China.

The cutoff 14 and 15 were found to be appropriate to differentiate people with clinical AN and BN from HC with sensitivity $(0.67 ; 0.79)$ and specificity $(0.82 ; 0.86)$ in our sample, respectively. According to the highest YI for the total EDs group, the optimal cutoff was 15(0.524), and the cutoff 14 and 15 had similar YIs. This suggests that the cutoff for clinical ED range between 14 and 15 as this will provide enough sensitivity and specificity to differentiate EDs patients from HC. Furthermore, all three areas under the ROC curves (AUCs) in the three groups were greater than 0.80, suggesting sufficient discriminatory ability of the EAT-26 in our clinical samples.

Despite the optimal cutoff points with acceptable AUCs in the current study, the results for sensitivity were not satisfactory. In general, the sensitivity found (0.663 0.793) was less acceptable than the specificity $(0.818 \sim 0.861)$ among the EDs, AN and BN groups. Different clinical cutoffs have provided different results regarding sensitivity, and specificity in the literature. For example, a clinical cut off of 20 from the English version has a sensitivity of 1.00 and specificity of 0.91 (King, 1986), a lower cutoff of 10 from the Arabic version of the EAT-26 has a sensitivity of 0.64 and a 
specificity of 0.38 (Al-Adawi et al., 2002), and a cutoff of 23from the Spanish version has a sensitivity of 0.60 and a specificity of 0.95 (Rivas et al., 2010).

Two possible explanations can be considered for the different findings of our study when comparing with previous reports. Firstly, the sample composition of prior studies was different in culture, age, gender, which may result in different values. For example, some surveys were conducted on females only while others in both genders (Al-Adawi et al., 2002, Nunes et al., 2005), and some samples consisted of patients with EDs as one group (King, 1986) while others focused on specific ED diagnosis and community controls (Siervo et al., 2005). Secondly, the social and culture difference between studies could play important roles in the inconsistency in their findings. The EAT-26 total scores of the EDs patients in our study (mean=23.70, $S D=16.00$ ) was smaller than those in Western patients (mean=31.23, $S D=12.39$ ) (Mintzet al., 2000). This may contribute to a smaller cutoff of the EAT-26 in our sample compared with the Western.

The distribution of total scores among subsamples (AN, BN, and $\mathrm{HC}$ ) was approximately equivalent to the data reported by Lee et al.(2002)(Lee et al., 2002), but differed from reported results from non-Asian populations (Garfinkel et al., 2001). People from China had lower total scores than non-Asians populations, perhaps because people from Asian are encouraged on a sociocultural basis to use denial and minimization in coping with psychiatric disorders (Lee \&Lock, 2007).

This study had several limitations, such as the fact that EDs people were older than HC, although this variable was controlled for, or the fact that only a small number of participants completed the retest and test-retest reliability examination. As the sample included young female 
participants only in view of the small number of male ED patients attending services during the study period(only 10). Therefore, the results may not be able to be generalized to male or older people with ED.

Despite the above limitations, a major and clear strength of the present study may be its clinical sample size. Our findings provided evidence that the Chinese version of the EAT-26 is a psychometrically reliable and valid self-rating instrument to identify people suffering from an ED in Mainland China. A clinical cutoff range between 14 and 15 could be used but caution should be exercise due to the low sensitivity. 


\section{References}

Agüera, Z., Brewin, N., Chen, J., Granero, R., Kang, Q., Fernandez-Aranda, F., \& Arcelus, J. (2017). Eating symptomatology and general psychopathology in patients with anorexia nervosa from China, UK and Spain: A cross-cultural study examining the role of social attitudes. PLoS One.12(3):e0173781.DOI:10.1371/journal.pone.0173781.

Al-Adawi, S., Dorvlo, A. S., Burke, D. T., Moosa,S.,\&Al-Bahlani, S. (2002). A survey of anorexia nervosa using the Arabic version of the EAT-26 and "gold standard" interviews among Omani adolescents. Eating and Weight Disorders, 7, 304-311.

APA (2000). Diagnostic and statistical manual of mental disorders DSM-IV-TR fourth edition (text revision). Washington, DC, American Psychiatric Association.

Brewin, N., Baggott, J., Dugard, P.,\& Arcelus,J. (2014). Clinical normative data for eating disorder examination questionnaire and eating disorder inventory for DSM-5 feeding and eating disorder classifications: a retrospective study of patients formerly diagnosed via DSM-IV. European Eating Disorders Review,22, 299-305. DOI: 10.1002/erv.2301.

Dolan, B. (1991). Cross-cultural aspects of anorexia nervosa and bulimia: A review. International Journal of Eating Disorders, 10(1), 67-79.

Garfinkel, P. E.\& Newman,A. (2001). The eating attitudes test: twenty-five years later. Eating and Weight Disorders, 6, 1-24.

Garner, D. M., Olmstead,M. P.,\& Polivy, J. (1983). Development and validation of a multidimensional eating disorder inventory for anorexia nervosa and bulimia. International Journal of Eating Disorders, 2, 15-34.

Garner, D. M., Olmsted, M. P., Bohr, Y.,\& Garfinkel, P. E. (1982). The eating attitudes test: psychometric features and clinical correlates. Psychological Medicine,12, 871-878.

Hudson, J. I., Hiripi, E., Pope, H. G., Jr., \& Kessler, R. C. (2007). The prevalence and correlates of eatingdisorders in the National Comorbidity Survey Replication. Biological Psychiatry, 61(3), 348358. DOI: 10.1016/j.biopsych.2006.03.040.

Huon, G. F., Qian, M. Y., Oliver, K., \& Xiao, G. L. (2002). A large-scale survey of eating disorder symptomatology among female adolescents in the People's Republic of China. International Journal of Eating Disorders, 32(2), 192-205. DOI: 10.1002/eat.10061.

King, M. B. (1986). Eating disorders in general practice. British Medical Journal, 293, 1412-1414.

Lee, H. Y. \&Lock,J. (2007). Anorexia nervosa in Asian-American adolescents: do they differ from their non-Asian peers. International Journal of Eating Disorders, 40, 227-231. DOI: 10.1002/eat.20364.

Lee, S., Kwok, K., Liau, C. \& Leung, T. (2002). Screening Chinese patients with eating disorders using the Eating Attitudes Test in Hong Kong. International Journal of Eating Disorders, 32, 91-97. DOI: 10.1002/eat.10064.

Lee, S. \& Lee, A. M. (2000). Disordered eating in three communities of China: a comparative study of female high school students in hong kong, Shenzhen, and rural hunan. International Journal of Eating Disorders, 27, 317-327.

Mintz, L. B.\& O'Halloran, M. S. (2000). The Eating Attitudes Test: validation with DSM-IV eating disorder criteria. Journal of Personality Assessment, 74, 489-503. DOI: 10.1207/s15327752jpa7403_11.

Nunes, M. A., Camey, S., Olinto, M. T. A.,\& Mari, J. d. J. (2005). The validity and 4-year test-retest reliability of the Brazilian version of the Eating Attitudes Test-26. Brazilian Journal of Medical and Biological Research, 38, 1655-1662. 
Pinninti, N. R., Madison, H., Musser, E., \& Rissmiller,D. (2003). MINI International Neuropsychiatric Schedule: clinical utility and patient acceptance. European Psychiatry, 18, 361-364.

Rivas, T., Bersabé, R., Jiménez, M.,\& Berrocal, C. (2010). The eating attitudes test (EAT-26): reliability and validity in Spanish female samples. The Spanish Journal of Psychology, 13, 1044-1056.

Si, T. M., Shu, L., Dang, W. M., Su, Y. A., Chen, J. X., Dong, W. T., Kong, Q. M., \&Zhang, W. H. (2009). Evaluation of the reliability and validity of Chinese version of the Mini-International Neuropsychiatric Interview in patients with mental disorders. Chinese Mental Health Journal, 23, 493-497,503. DOI: 10.3969/j.issn.1000-6729.2009.07.011.

Siervo, M., Boschi,V., Papa,A., Bellini,O.,\& Falconi,C. (2005). Application of the SCOFF, Eating Attitude Test 26 (EAT 26) and Eating Inventory (TFEQ) Questionnaires in young women seeking diet-therapy. Eating and Weight Disorders, 10, 76-82.

Swets, J. A. (1988). Measuring the accuracy of diagnostic systems. Science, 240, 1285-1293.

Tong, J., Shi, J., Wang, J., Zhang, H., Zhang, S. F, Wu, X. Y., \&Hsu, L.K. (2014). A two-stage epidemiologic study on prevalence of eating disorders in female university students in Wuhan, China. Social Psychiatry and Psychiatric Epidemiology, 49, 499-505. DOI: 10.1007/s00127-0130694-y.

Weir, J. P. (2005). Quantifying test-retest reliability using the intraclass correlation coefficient and the SEM. Journal of Strength and Conditioning Research,19, 231-240. DOI: 10.1519/15184.1.

Zhang, W. H., Zhang, D. R., \& Qian, Y. (2006). Psychological characteristics of patients with eating disorders. Chinese Mental Health Journal, 20, 596-599. 
Table 1.The demographic and clinical characteristics of all participants $(\mathrm{n}=802)$

\begin{tabular}{|c|c|c|c|c|c|c|c|}
\hline & \multicolumn{6}{|c|}{ mean (SD) } & \multirow{2}{*}{ All ED vs. $\mathrm{HC}(\mathrm{t} / p)$} \\
\hline & All ED & AN & $\mathrm{BN}$ & BED & OSFED & $\mathrm{HC}$ & \\
\hline $\mathrm{n}$ & 396 & 240 & 84 & 28 & 44 & 406 & \\
\hline Age(years) & $19.62(4.53)$ & $18.83(4.37)$ & $22.46(5.38)$ & $19.67(5.30)$ & $18.95(4.35)$ & $18.35(2.67)$ & $5.009^{* * *}$ \\
\hline Onset age(years) & 16.93(3.06) & $16.50(2.84)$ & $18.73(4.18)$ & $16.94(2.69)$ & $15.48(2.99)$ & -- & -- \\
\hline DOE(months) & $32.24(34.20)$ & $26.77(29.40)$ & $42.73(37.12)$ & $53.59(52.89)$ & $39.38(49.82)$ & -- & -- \\
\hline BMI & $16.24(3.09)$ & $15.28(2.11)$ & $19.52(2.49)$ & $20.74(2.52)$ & $14.79(2.30)$ & $20.25(2.74)$ & $-18.760 * *$ \\
\hline EAT-26 & $23.70(16.00)$ & $22.65(15.87)$ & $31.04(14.52)$ & $17.04(14.06)$ & $13.81(10.42)$ & $7.22(7.85)$ & $20.109 * *$ \\
\hline
\end{tabular}

Note: $* * p<0.001 . \mathrm{AN}=$ anorexia nervosa, $\mathrm{AN}-\mathrm{R}=$ anorexia nervosa of restricting subtype, $\mathrm{AN}-\mathrm{BP}=$ anorexia nervosa of bulimia/ purging subtype, $\mathrm{BMI}=$ body mass index, $\mathrm{BN}=$ bulimia nervosa, $\mathrm{DOE}=$ Duration of eating disorder, $\mathrm{ED}=$ eating disorder, $\mathrm{EAT}-26=$ total score of twenty-six items eating attitude test, $\mathrm{HC}=$ healthy control, $\mathrm{OSFED}=$ other specified feeding or eating disorder 
Table 2. The ROC analysis for the cutoffs, sensitivity, specificity and Youden index of the total EAT-26 scores for the ED, AN, and BN groups

\begin{tabular}{|c|c|c|c|c|c|c|c|c|c|c|c|c|c|c|c|}
\hline & Cutoffs & 10 & 11 & 12 & 13 & 14 & 15 & 16 & 17 & 18 & 19 & 20 & 21 & 22 & 23 \\
\hline \multirow{4}{*}{ EDs } & Sensitivity & 0.770 & 0.760 & 0.727 & 0.699 & 0.676 & $\mathbf{0 . 6 6 3}^{1}$ & 0.643 & 0.615 & 0.589 & 0.561 & 0.526 & 0.500 & 0.477 & 0.454 \\
\hline & & & & & & & & & & & & & & & \\
\hline & Specificity & 0.731 & 0.763 & 0.786 & 0.816 & 0.847 & 0.861 & 0.879 & 0.888 & 0.897 & 0.907 & 0.918 & 0.923 & 0.932 & 0.939 \\
\hline & YI & 0.501 & 0.523 & 0.513 & 0.515 & 0.523 & 0.524 & 0.522 & 0.502 & 0.486 & 0.469 & 0.444 & 0.423 & 0.409 & 0.393 \\
\hline \multirow{3}{*}{ AN } & Sensitivity & 0.763 & 0.755 & 0.727 & 0.691 & 0.667 & 0.651 & 0.631 & 0.610 & 0.574 & 0.534 & 0.490 & 0.466 & 0.434 & 0.418 \\
\hline & Specificity & 0.704 & 0.736 & 0.760 & 0.789 & 0.818 & 0.832 & 0.851 & 0.860 & 0.870 & 0.880 & 0.892 & 0.897 & 0.906 & 0.916 \\
\hline & YI & 0.467 & 0.491 & 0.487 & 0.480 & 0.485 & 0.483 & 0.482 & 0.470 & 0.444 & 0.414 & 0.382 & 0.363 & 0.340 & 0.334 \\
\hline \multirow{3}{*}{$\mathrm{BN}$} & Sensitivity & 0.901 & 0.883 & 0.847 & 0.829 & 0.802 & 0.793 & 0.775 & 0.721 & 0.712 & 0.712 & 0.694 & 0.658 & 0.649 & 0.613 \\
\hline & Specificity & 0.731 & 0.763 & 0.786 & 0.816 & 0.847 & 0.861 & 0.879 & 0.888 & 0.897 & 0.907 & 0.918 & 0.923 & 0.932 & 0.939 \\
\hline & YI & 0.632 & 0.646 & 0.633 & 0.645 & 0.649 & 0.654 & 0.654 & 0.608 & 0.608 & 0.619 & 0.612 & 0.581 & 0.581 & 0.552 \\
\hline
\end{tabular}

Note: ${ }^{1}$ : All letters marked in bold means the best-fitting cutoffs according to the Youden indices. 